\title{
HOMOLOGICAL DIMENSIONS WITH RESPECT TO A SEMIDUALIZING COMPLEX
}

\author{
JONATHAN TOTUSHEK
}

\begin{abstract}
In this paper, we build off of Takahashi and White's $\mathcal{P}_{C}$-projective dimension and $\mathcal{I}_{C}$-injective dimension to define these dimensions for when $C$ is a semidaulizing complex. We develop the framework for these homological dimensions by establishing base change results and localglobal behavior. Furthermore, we investigate how these dimensions interact with other invariants.
\end{abstract}

1. Introduction. Let $R$ be a commutative Noetherian ring. The projective, flat, and injective dimensions of an $R$-module $M$ are now classical invariants that are important for studying $M$ and $R$. These dimensions were later generalized for $R$-complexes by Foxby [3], and many useful results about dimensions for modules also hold true for complexes.

A finitely generated $R$-module $C$ is semidualizing if $R \cong \operatorname{Hom}_{R}(C, C)$ and $\operatorname{Ext}_{R}^{\geq 1}(C, C)=0$. Takahashi and White [10] defined, for a semidualizing $R$-module $C$, the $\mathcal{P}_{C}$-projective and $\mathcal{I}_{C}$-injective dimensions. The $\mathcal{P}_{C}$-projective dimension of an $R$-module $M\left(\mathcal{P}_{C}-\operatorname{pd}_{R}(M)\right)$ is the length of the shortest resolution of $M$ by modules of the form $C \otimes_{R} P$ where $P$ is a projective module. They define $\mathcal{I}_{C}$-injective dimension $\left(\mathcal{I}_{C}\right.$-id $\left.R(M)\right)$ dually, and one defines the $\mathcal{F}_{C}$-projective dimension $\left(\mathcal{F}_{C}-\mathrm{pd}_{R}(M)\right)$ similarly. We extend these constructions to the realm of $R$-complexes. Note that we work in the derived category $\mathcal{D}(R)$. See Section 2 for some background and notation on this subject.

A complex $C \in \mathcal{D}_{\mathrm{b}}^{\mathrm{f}}(R)$ is semidualizing if the natural homothety morphism $\chi_{C}^{R}: R \rightarrow \mathbf{R H o m}_{R}(C, C)$ is an isomorphism in $\mathcal{D}(R)$. An $R$-complex $D$ is dualizing if it is semidualizing and has finite injective dimension. To understand the $\mathcal{P}_{C}$-projective, $\mathcal{F}_{C}$-projective and $\mathcal{I}_{C^{-}}$

2010 AMS Mathematics subject classification. Primary 13D02, 13D05, 13D09.

Keywords and phrases. Auslander class, Bass class, flat dimension, injective dimension, projective dimension, semidualizing complex.

Received by the editors on November 24, 2014. 
injective dimensions in this context, we use the following result, see Theorem 3.9 below.

Theorem 1.1. Let $X \in \mathcal{D}_{\mathrm{b}}(R)$, and let $C$ be a semidualizing $R$ complex.

(i) We have $\operatorname{pd}_{R}\left(\mathbf{R H o m}_{R}(C, X)\right)<\infty$ if and only if there exists $Y \in \mathcal{D}_{\mathrm{b}}(R)$ such that $\operatorname{pd}_{R}(Y)<\infty$ and $X \simeq C \otimes_{R}^{\mathbf{L}} Y$ in $\mathcal{D}(R)$. When these conditions are satisfied, one has $Y \simeq \mathbf{R H o m}_{R}(C, X)$ and $X \in \mathcal{B}_{C}(R)$.

(ii) We have $\mathrm{fd}_{R}\left(\mathbf{R H o m}_{R}(C, X)\right)<\infty$ if and only if there exists $Y \in \mathcal{D}_{\mathrm{b}}(R)$ such that $\operatorname{fd}_{R}(Y)<\infty$ and $X \simeq C \otimes_{R}^{\mathbf{L}} Y$ in $\mathcal{D}(R)$. When these conditions are satisfied, one has $Y \simeq \mathbf{R H o m}_{R}(C, X)$ and $X \in \mathcal{B}_{C}(R)$.

(iii) We have $\operatorname{id}_{R}\left(C \otimes_{R}^{\mathbf{L}} X\right)<\infty$ if and only if there exists $Y \in \mathcal{D}_{\mathrm{b}}(R)$ such that $\operatorname{id}_{R}(Y)<\infty$ and $X \simeq \mathbf{R H o m}_{R}(C, Y)$ in $\mathcal{D}(R)$. When these conditions are satisfied, one has $Y \simeq C \otimes_{R}^{\mathrm{L}} X$ and $X \in$ $\mathcal{A}_{C}(R)$.

With this in mind, we define, e.g., $\mathcal{P}_{C^{-}} \operatorname{pd}_{R}(X):=\sup (C)+$ $\operatorname{pd}_{R}\left(\mathbf{R} \operatorname{Hom}_{R}(C, X)\right)$; thus, $\mathcal{P}_{C^{-}} \operatorname{pd}_{R}(X)<\infty$ if and only if $X$ satisfies the equivalent conditions of Theorem 1.1 (i). We define $\mathcal{F}_{C^{-}} \operatorname{pd}_{R}(X)$ and $\mathcal{I}_{C^{-}} \operatorname{id}_{R}(X)$ similarly.

In Section 3 we develop the foundations of these homological dimensions. For instance, we establish finite flat dimension base change (3.11) and local-global principles (3.16)-(3.18). Also, in Theorem 3.10, we show how these notions naturally augment Foxby equivalence. In Section 4, we establish some stability results and the following, see Theorem 4.9 .

Theorem 1.2. Assume $R$ has a dualizing complex $D$, and let $X \in$

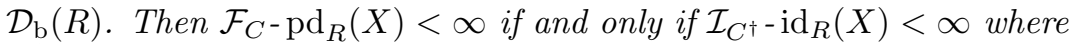
$C^{\dagger}=\mathbf{R H o m}_{R}(C, D)$.

This result is key for the work in [9].

2. Background. Throughout this paper, $R$ and $S$ are commutative Noetherian rings with identity, and $C$ is a semidualizing $R$-complex. 
We work in the derived category $\mathcal{D}(R)$ of complexes of $R$-modules, indexed homologically (see, e.g., $[\mathbf{5}, \mathbf{6}]$ ). A complex $X \in \mathcal{D}(R)$ is homologically bounded if $\mathrm{H}_{i}(X)=0$ for all $|i| \gg 0$ and $X$ is homologically finite if $\oplus_{i} \mathrm{H}_{i}(X)$ is finitely generated. We denote by $\mathcal{D}_{\mathrm{b}}(R)$ and $\mathcal{D}_{\mathrm{b}}^{\mathrm{f}}(R)$ the full subcategories of $\mathcal{D}(R)$ consisting of all homologically bounded $R$-complexes and all homologically finite $R$ complexes, respectively. Isomorphisms in $\mathcal{D}(R)$ are identified by the symbol $\simeq$.

For $R$-complexes $X$ and $Y$, let $\inf (X)$ and $\sup (X)$ denote the infimum and supremum, respectively, of the set $\left\{i \in \mathbb{Z} \mid \mathrm{H}_{i}(X)=0\right\}$. Let $X \otimes_{R}^{\mathbf{L}} Y$ and $\mathbf{R} \operatorname{Hom}_{R}(X, Y)$ denote the left-derived tensor product and right-derived homomorphism complexes, respectively.

Definition 2.1. Let $X \in \mathcal{D}_{+}(R)$. The projective dimension of $X$ is $\operatorname{pd}_{R}(X)=\inf \left\{\begin{array}{l|l}n \in \mathbb{Z} & \begin{array}{l}P \stackrel{\simeq}{\longrightarrow} X \text { where } P \text { is a complex of projective } \\ R \text {-modules such that } P_{i}=0 \text { for all } i>n\end{array}\end{array}\right\}$.

The flat dimension (fd) and injective dimension (id) are defined similarly. Let $\mathcal{P}(R), \mathcal{F}(R)$ and $\mathcal{I}(R)$ denote the full subcategories of $\mathcal{D}_{\mathrm{b}}(R)$ consisting of complexes of finite projective, flat and injective dimensions, respectively.

Fact 2.2 ([1, Proposition 4.5]). Let $X, Y \in \mathcal{D}(R)$.

(a) If $\operatorname{id}_{R}(Y)<\infty$, then $\operatorname{fd}_{R}\left(\operatorname{RHom}_{R}(X, Y)\right) \leq \operatorname{id}_{R}(X)+\sup (Y)$.

(b) If $\operatorname{fd}_{R}(Y)<\infty$, then $\operatorname{id}_{R}\left(X \otimes{ }_{R}^{\mathbf{L}} Y\right) \leq \operatorname{id}_{R}(X)-\inf (Y)$.

The following result is for use in Section 4.

Lemma 2.3. Let $X \in \mathcal{D}_{\mathrm{b}}(R)$.

(i) If I is a faithfully injective $R$-module and $\operatorname{id}_{R}\left(\operatorname{RHom}_{R}(X, E)\right) \leq$ $n$, then $\operatorname{fd}_{R}(X) \leq n$.

(ii) If $F$ is a faithfully flat $R$-module and $\operatorname{fd}_{R}\left(X \otimes_{R}^{\mathbf{L}} F\right) \leq n$, then $\mathrm{fd}_{R}(X) \leq n$.

(iii) If $E$ is a faithfully injective $R$-module and $\operatorname{id}_{R}(X) \leq n$, then we have that $\operatorname{fd}_{R}\left(\mathbf{R H o m}_{R}(X, E)\right) \leq n$. 
(iv) If $F$ is a faithfully flat $R$-module and $\operatorname{id}_{R}(X) \leq n$, then we have that $\operatorname{id}_{R}\left(X \otimes_{R}^{\mathbf{L}} E\right) \leq n$.

Proof.

(i) Assume that $\operatorname{id}_{R}\left(\mathbf{R H o m}_{R}(X, E)\right) \leq n$, and let $F \stackrel{\widetilde{ }}{\rightarrow} X$ be a flat resolution of $X$.

A standard truncation argument shows that $\operatorname{Hom}_{R}\left(\operatorname{Coker}\left(\partial_{n+1}^{F}\right), E\right)$ is injective. Since $E$ is faithfully injective, we also have that $\operatorname{Coker}\left(\partial_{n+1}^{F}\right)$ is flat. Thus, $\operatorname{fd}_{R}(X) \leq n$.

The proofs of (ii), (iii) and (iv) are similar.

Fact 2.4 ([1, Lemma 4.4]). Let $L, M, N \in \mathcal{D}(R)$. Assume that $L \in \mathcal{D}_{+}^{\mathrm{f}}(R)$.

The natural tensor-evaluation morphism

$$
\omega_{L M N}: \mathbf{R H o m}_{R}(L, M) \otimes_{R}^{\mathbf{L}} N \longrightarrow \mathbf{R H o m}_{R}\left(L, M \otimes_{R}^{\mathbf{L}} N\right)
$$

is an isomorphism when $M \in \mathcal{D}_{-}(R)$ and either $L \in \mathcal{P}(R)$ or $N \in$ $\mathcal{F}(R)$.

The natural Hom-evaluation morphism

$$
\theta_{L M N}: L \otimes{ }_{R}^{\mathbf{L}} \mathbf{R} \operatorname{Hom}_{R}(M, N) \longrightarrow \mathbf{R H o m}_{R}\left(\mathbf{R H o m}_{R}(L, M), N\right)
$$

is an isomorphism when $M \in \mathcal{D}_{\mathrm{b}}(R)$ and either $L \in \mathcal{P}(R)$ or $N \in \mathcal{I}(R)$.

Definition 2.5 (Foxby classes).

(i) The Auslander class with respect to $C$ is the full subcategory $\mathcal{A}_{C}(R) \subseteq \mathcal{D}_{\mathrm{b}}(R)$ such that a complex $X$ is in $\mathcal{A}_{C}(R)$ if and only if $C \otimes_{R}^{\mathbf{L}} X \in \mathcal{D}_{\mathrm{b}}(R)$ and the natural morphism $\gamma_{X}^{C}: X \rightarrow$ $\mathbf{R} \operatorname{Hom}_{R}\left(C, C \otimes \otimes_{R}^{\mathbf{L}} X\right)$ is an isomorphism in $\mathcal{D}(R)$.

(ii) The Bass class with respect to $C$ is the full subcategory $\mathcal{B}_{C}(R) \subseteq$ $\mathcal{D}_{\mathrm{b}}(R)$ such that a complex $Y$ is in $\mathcal{B}_{C}(R)$ if and only if $\mathbf{R H o m}_{R}(C, Y) \in \mathcal{D}_{\mathrm{b}}(R)$ and the natural morphism $\xi_{Y}^{C}: C \otimes \mathbb{L}_{R}$ $\mathbf{R H o m}_{R}(C, Y) \rightarrow Y$ is an isomorphism in $\mathcal{D}(R)$.

For a generalized diagrammatic version of the next result, see Theorem 3.10. 
Fact 2.6 (Foxby equivalence [2, Theorem 4.6]). Let $X, Y \in \mathcal{D}_{\mathrm{b}}(R)$. We have that

(a) $X \in \mathcal{A}_{C}(R)$ if and only if $C \otimes_{R}^{\mathbf{L}} X \in \mathcal{B}_{C}(R)$.

(b) $Y \in \mathcal{B}_{C}(R)$ if and only if $\mathbf{R H o m}_{R}(C, Y) \in \mathcal{A}_{C}(R)$.

Fact 2.7 ([2, Proposition 4.4]). Let $X \in \mathcal{D}_{\mathrm{b}}(R)$.

(a) If $\operatorname{fd}_{R}(X)<\infty$ (e.g., $\operatorname{pd}_{R}(X)<\infty$ ), then $X \in \mathcal{A}_{C}(R)$.

(b) If $\operatorname{id}_{R}(X)<\infty$, then $X \in \mathcal{B}_{C}(R)$.

3. $C$-Dimensions for complexes. In this section, we define the $\mathcal{P}_{C}$-projective, $\mathcal{F}_{C}$-projective and $\mathcal{I}_{C}$-injective dimensions and build their foundations.

Definition 3.1. Let $X \in \mathcal{D}_{\mathrm{b}}(R)$.

(i) The $\mathcal{P}_{C}$-projective dimension of $X$ is defined as

$$
\mathcal{P}_{C^{-}} \operatorname{pd}_{R}(X)=\sup (C)+\operatorname{pd}_{R}\left(\mathbf{R H o m}_{R}(C, X)\right) .
$$

(ii) The $\mathcal{F}_{C}$-projective dimension of $X$ is defined as

$$
\mathcal{F}_{C^{-}} \operatorname{pd}_{R}(X)=\sup (C)+\mathrm{fd}_{R}\left(\mathbf{R} \operatorname{Hom}_{R}(C, X)\right) .
$$

(iii) The $\mathcal{I}_{C}$-injective dimension of $X$ is defined as

$$
\mathcal{I}_{C^{-}} \operatorname{id}_{R}(X)=\sup (C)+\operatorname{id}_{R}\left(C \otimes_{R}^{\mathbf{L}} X\right) .
$$

Let $\mathcal{P}_{C}(R), \mathcal{F}_{C}(R)$, and $\mathcal{I}_{C}(R)$ denote the full subcategories of $\mathcal{D}_{\mathrm{b}}(R)$ of all complexes of finite $C$-projective, $C$-flat, and $C$-injective dimension, respectively.

Remark 3.2. Let $X \in \mathcal{D}_{\mathrm{b}}(R)$. Observe that $\sup (C)<\infty$. Hence, $\mathcal{P}_{C^{-}} \operatorname{pd}_{R}(X)<\infty$ if and only if $\operatorname{pd}_{R}\left(\mathbf{R H o m}_{R}(C, X)\right)<\infty$. If $\mathcal{P}_{C^{-}} \operatorname{pd}_{R}(X)<\infty$, then Fact 2.7 (a) implies that $\mathbf{R H o m}_{R}(C, X) \in$ $\mathcal{A}_{C}(R)$ and Foxby equivalence (2.6) implies that $X \in \mathcal{B}_{C}(R)$. Similarly, $\mathcal{F}_{C^{-}} \operatorname{pd}_{R}(X)<\infty$ if and only if $\mathrm{fd}_{R}\left(\mathbf{R H o m}_{R}(C, X)\right)<\infty$. If $\mathcal{F}_{C^{-}} \operatorname{pd}_{R}(X)<\infty$, then $X \in \mathcal{B}_{C}(R)$. Also, we have $\mathcal{I}_{C^{-}} \mathrm{id}_{R}(X)<\infty$ if and only if $\operatorname{id}_{R}\left(C \otimes_{R}^{\mathbf{L}} X\right)<\infty$. Hence, if $\mathcal{I}_{C}-\mathrm{id}_{R}(X)<\infty$, then $X \in \mathcal{A}_{C}(R)$. 
Remark 3.3. Let $X \in \mathcal{D}_{\mathrm{b}}(R)$. Note that, when $C=R$, we have that $\left.\mathcal{P}_{C^{-}-\operatorname{pd}_{R}(X)=\sup (R)+\operatorname{pd}_{R}(\mathbf{R H o m}}(R, X)\right)=\operatorname{pd}_{R}(X)$. Similarly, in this case, $\mathcal{F}_{C^{-}} \operatorname{pd}_{R}(X)=\mathrm{fd}_{R}(X)$ and $\mathcal{I}_{C^{-}} \operatorname{id}_{R}(X)=\operatorname{id}_{R}(X)$.

Remark 3.4. Let $M$ be an $R$-module. When $C$ is a semidualizing $R$-module, Takahashi and White [10, Theorem 2.11], using the definition described in Section 1, showed that $\mathcal{P}_{C}-\operatorname{pd}_{R}(X)=$ $\operatorname{pd}_{R}\left(\mathbf{R H o m}_{R}(C, X)\right)$. Since $\sup (C)=0$ in this case, Definition 3.1 (i) shows that our definition is consistent with the one from [10]. In a similar way, it can be shown that $\mathcal{I}_{C}$-id recovers Takahashi and White's definition in this case.

The next result compares $\mathcal{F}_{C^{-}}$pd with $\mathcal{P}_{C^{-}}$pd.

Proposition 3.5. Let $X \in \mathcal{D}_{\mathrm{b}}(R)$. Then

$$
\mathcal{F}_{C}-\operatorname{pd}_{R}(X) \leq \mathcal{P}_{C}-\operatorname{pd}_{R}(X) \leq \mathcal{F}_{C}-\operatorname{pd}_{R}(X)+\operatorname{dim}(R) .
$$

In particular, if $\operatorname{dim}(R)<\infty$, then we have $\mathcal{P}_{C}-\operatorname{pd}_{R}(X)<\infty$ if and only if $\mathcal{F}_{C}-\operatorname{pd}_{R}(X)<\infty$.

Proof. Assume that $\mathcal{P}_{C^{-}} \operatorname{pd}_{R}(X)=n<\infty$. Then

$$
\mathrm{fd}_{R}\left(\mathbf{R H o m}_{R}(C, X)\right) \leq \operatorname{pd}_{R}\left(\mathbf{R H o m}_{R}(C, X)\right)=n-\sup (C)<\infty .
$$

It now follows that $\mathcal{F}_{C}-\operatorname{pd}_{R}(X) \leq n$.

Next assume that $\operatorname{dim}(R)<\infty$ and $\mathcal{F}_{C^{-}} \operatorname{pd}_{R}(X)=n<\infty$. By [8], we have

$$
\begin{aligned}
\operatorname{pd}_{R}\left(\mathbf{R H o m}_{R}(C, X)\right) & \leq \mathrm{fd}_{R}\left(\mathbf{R H o m}_{R}(C, X)\right)+\operatorname{dim}(R) \\
& =n-\sup (C)+\operatorname{dim}(R) .
\end{aligned}
$$

Therefore, $\mathcal{P}_{C^{-}} \operatorname{pd}_{R}(X) \leq \operatorname{dim}(R)+n$.

The following three results are versions of [10, Theorem 2.11] involving a semidaulizing complex.

Proposition 3.6. Let $X \in \mathcal{D}_{\mathrm{b}}(R)$. Then, we have

$$
\mathcal{P}_{C}-\operatorname{pd}_{R}\left(C \otimes_{R}^{\mathbf{L}} X\right)=\sup (C)+\operatorname{pd}_{R}(X) .
$$

In particular, $\mathcal{P}_{C}-\operatorname{pd}_{R}\left(C \otimes_{R}^{\mathrm{L}} X\right)<\infty$ if and only if $\operatorname{pd}_{R}(X)<\infty$. 
Proof. Let $n \in \mathbb{Z}$. We prove that $\mathcal{P}_{C^{-}} \operatorname{pd}_{R}\left(C \otimes_{R}^{\mathbf{L}} X\right) \leq n$ if and only if $\sup (C)+\operatorname{pd}_{R}(X) \leq n$.

For the forward implication, assume that $\mathcal{P}_{C^{-}} \operatorname{pd}_{R}\left(C \otimes_{R}^{\mathrm{L}} X\right) \leq n$. Then, by Definition 3.1 (i), we have

$$
\sup (C)+\operatorname{pd}_{R}\left(\mathbf{R H o m}_{R}\left(C, C \otimes_{R}^{\mathbf{L}} X\right)\right)=\mathcal{P}_{C^{-}} \operatorname{pd}_{R}\left(C \otimes_{R}^{\mathbf{L}} X\right) \leq n .
$$

Thus, $\operatorname{pd}_{R}\left(\mathbf{R H o m}_{R}\left(C, C \otimes_{R}^{\mathbf{L}} X\right)\right)<\infty$. Fact 2.7 (a) implies $\mathbf{R H o m}_{R}(C$, $\left.C \otimes_{R}^{\mathbf{L}} X\right) \in \mathcal{A}_{C}(R)$. By Foxby equivalence (2.6), we have $C \otimes_{R}^{\mathbf{L}} X \in$ $\mathcal{B}_{C}(R)$ and $X \in \mathcal{A}_{C}(R)$. Therefore, we have $X \simeq \mathbf{R H o m}_{R}\left(C, C \otimes \mathbb{L}_{R} X\right)$ and $\operatorname{pd}_{R}\left(\mathbf{R} \operatorname{Hom}_{R}\left(C, C \otimes_{R}^{\mathbf{L}} X\right)\right)=\operatorname{pd}_{R}(X)$. Thus, $\sup (C)+\operatorname{pd}_{R}(X) \leq$ $n$.

For the reverse implication, assume that $\sup (C)+\operatorname{pd}_{R}(X) \leq$ $n$. In particular, we have that $\operatorname{pd}_{R}(X)<\infty$. Therefore, $X \in$ $\mathcal{A}_{C}(R)$ and $X \simeq \mathbf{R H o m}_{R}\left(C, C \otimes_{R}^{\mathbf{L}} X\right)$. It follows that $\operatorname{pd}_{R}(X)=$ $\operatorname{pd}_{R}\left(\mathbf{R H o m}_{R}\left(C, C \otimes_{R}^{\mathbf{L}} X\right)\right)$. By Definition 3.1 (i), we have

$$
\begin{aligned}
\mathcal{P}_{C^{-}} \operatorname{pd}_{R}\left(C \otimes_{R}^{\mathbf{L}} X\right) & =\sup (C)+\operatorname{pd}_{R}\left(\mathbf{R} \operatorname{Hom}_{R}\left(C, C \otimes_{R}^{\mathbf{L}} X\right)\right) \\
& =\sup (C)+\operatorname{pd}_{R}(X) \leq n .
\end{aligned}
$$

The next two results are proven like Proposition 3.6.

Proposition 3.7. Let $X \in \mathcal{D}_{\mathrm{b}}(R)$. Then we have

$$
\mathcal{F}_{C}-\operatorname{pd}_{R}\left(C \otimes_{R}^{\mathbf{L}} X\right)=\sup (C)+\operatorname{fd}_{R}(X) .
$$

In particular, $\mathcal{F}_{C}-\operatorname{pd}_{R}\left(C \otimes_{R}^{\mathbf{L}} X\right)<\infty$ if and only if $\operatorname{fd}_{R}(X)<\infty$.

Proposition 3.8. Let $X \in \mathcal{D}_{\mathrm{b}}(R)$. Then we have

$$
\mathcal{I}_{C}-\operatorname{id}_{R}\left(\mathbf{R H o m}_{R}(C, X)\right)=\sup (C)+\operatorname{id}_{R}(X) .
$$

In particular, $\mathcal{I}_{C}-\operatorname{id}_{R}\left(\mathbf{R H o m}_{R}(C, X)\right)<\infty$ if and only if $\operatorname{id}_{R}(X)<\infty$.

Next, we have Theorem 1.1 from the introduction.

Theorem 3.9. Let $X \in \mathcal{D}_{\mathrm{b}}(R)$.

(i) We have $\mathcal{P}_{C}-\operatorname{pd}_{R}(X)<\infty$ if and only if there exists $Y \in \mathcal{D}_{\mathrm{b}}(R)$ such that $\operatorname{pd}_{R}(Y)<\infty$ and $X \simeq C \otimes_{R}^{\mathbf{L}} Y$. When these conditions are satisfied, one has $Y \simeq \mathbf{R H o m}_{R}(C, X)$ and $X \in \mathcal{B}_{C}(R)$. 
(ii) We have $\mathcal{F}_{C}-\operatorname{pd}_{R}(X)<\infty$ if and only if there exists $Y \in \mathcal{D}_{\mathrm{b}}(R)$ such that $\operatorname{fd}_{R}(Y)<\infty$ and $X \simeq C \otimes_{R}^{\mathrm{L}} Y$. When these conditions are satisfied, one has $Y \simeq \mathbf{R H o m}_{R}(C, X)$ and $X \in \mathcal{B}_{C}(R)$.

(iii) We have $\mathcal{I}_{C}-\operatorname{id}_{R}(X)<\infty$ if and only if there exists $Y \in \mathcal{D}_{\mathrm{b}}(R)$ such that $\operatorname{id}_{R}(Y)<\infty$ and $X \simeq \mathbf{R H o m}_{R}(C, Y)$. When these conditions are satisfied, one has $Y \simeq C \otimes_{R}^{\mathrm{L}} X$ and $X \in \mathcal{A}_{C}(R)$.

Proof.

(i) For the forward implication, assume that $\mathcal{P}_{C}-\operatorname{pd}_{R}(X)<\infty$. Then, by Definition 3.1 (i), we have $\operatorname{pd}_{R}\left(\mathbf{R H o m}_{R}(C, X)\right)=\mathcal{P}_{C^{-}}$ $\operatorname{pd}_{R}(X)-\sup (C)<\infty$. Fact 2.7 (a) implies that $\mathbf{R} \operatorname{Hom}_{R}(C, X)$ $\in \mathcal{A}_{C}(R)$ and Foxby equivalence implies that $X \in \mathcal{B}_{C}(R)$.

Thus, $X \simeq C \otimes_{R}^{\mathbf{L}} \mathbf{R H o m}_{R}(C, X) \simeq C \otimes_{R}^{\mathbf{L}} Y$ with $Y=\mathbf{R H o m}_{R}(C, X)$.

For the reverse implication, assume that there exists a $Y \in \mathcal{D}_{\mathrm{b}}(R)$ such that $\operatorname{pd}_{R}(Y)<\infty$ and $X \simeq C \otimes_{R}^{\mathrm{L}} Y$. Then Fact 2.7 (a) implies that $Y \in \mathcal{A}_{C}(R)$, and hence we have

$$
Y \simeq \mathbf{R H o m}_{R}\left(C, C \otimes_{R}^{\mathbf{L}} Y\right) \simeq \mathbf{R H o m}_{R}(C, X) .
$$

It now follows from Definition 3.1 (i) that $\mathcal{P}_{C^{-}} \operatorname{pd}_{R}(X)<\infty$.

Parts (ii) and (iii) are proven similarly.

The previous results give rise to a generalized Foxby equivalence.

Theorem 3.10 (Foxby equivalence). There is a commutative diagram:

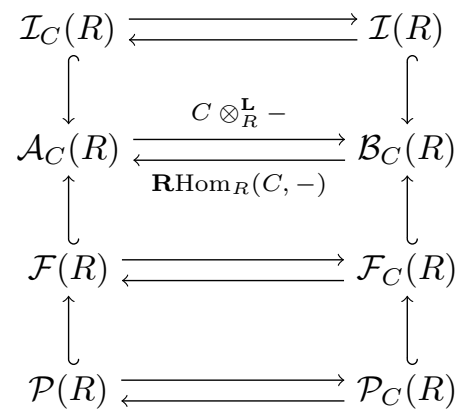


where the vertical arrows are full embeddings, and the unlabeled horizontal arrows are quasi-inverse equivalences of categories.

The next result shows how $\mathcal{P}_{C^{-}}$pd and $\mathcal{F}_{C^{-}}$pd transfer along a ring homomorphism of finite flat dimension. Note that, if $\varphi: R \rightarrow S$ is a ring homomorphism of finite flat dimension, then $C \otimes{ }_{R}^{\mathbf{L}} S$ is a semidualizing $S$-complex by $[\mathbf{2}$, Theorem 5.6] and [4, Theorem II (a)].

Proposition 3.11. Let $\varphi: R \rightarrow S$ be a ring homomorphism of finite flat dimension and $X \in \mathcal{D}_{\mathrm{b}}(R)$. Then we have:

(i) $\mathcal{P}_{C \otimes \otimes_{R} S}-\operatorname{pd}_{S}\left(X \otimes_{R}^{\mathrm{L}} S\right)-\sup \left(C \otimes_{R}^{\mathrm{L}} S\right) \leq \mathcal{P}_{C}-\operatorname{pd}_{R}(X)-\sup (C)$,

(ii) $\mathcal{F}_{C \otimes_{R}^{\mathrm{L}} S^{-}} \operatorname{pd}_{S}\left(X \otimes_{R}^{\mathrm{L}} S\right)-\sup \left(C \otimes_{R}^{\mathrm{L}} S\right) \leq \mathcal{F}_{C}-\operatorname{pd}_{R}(X)-\sup (C)$,

(iii) $\mathcal{P}_{C \otimes} \mathbb{L}_{R}-\operatorname{pd}_{S}\left(X \otimes{ }_{R}^{\mathrm{L}} S\right) \leq \mathcal{P}_{C}-\operatorname{pd}_{R}(X)$, and

(iv) $\mathcal{F}_{C \otimes} \mathbb{L}_{S}-\operatorname{pd}_{S}\left(X \otimes_{R}^{\mathrm{L}} S\right) \leq \mathcal{F}_{C}-\operatorname{pd}_{R}(X)$.

Equality holds when $\varphi$ is faithfully flat.

Proof. (i) and (iii). Assume first that $\mathcal{P}_{C}-\operatorname{pd}_{R}(X)-\sup (C)=n<$ $\infty$. Then $\operatorname{pd}_{R}\left(\mathbf{R H o m}_{R}(C, X)\right)=n$, and hence, by the base change, we have

$$
\operatorname{pd}_{S}\left(\mathbf{R H o m}_{R}(C, X) \otimes_{R}^{\mathbf{L}} S\right) \leq \operatorname{pd}_{R}(\mathbf{R H o m}(C, X))=n .
$$

Observe that, by tensor-evaluation (2.4) and Hom-tensor adjointness, there are isomorphisms

$$
\begin{aligned}
\mathbf{R H o m}_{R}(C, X) \otimes_{R}^{\mathbf{L}} S & \simeq \mathbf{R H o m}_{R}\left(C, X \otimes_{R}^{\mathbf{L}} S\right) \\
& \simeq \mathbf{R} \operatorname{Hom}_{R}\left(C, \mathbf{R} H_{0}\left(S, X \otimes_{R}^{\mathbf{L}} S\right)\right) \\
& \simeq \mathbf{R H o m}_{S}\left(C \otimes_{R}^{\mathbf{L}} S, X \otimes_{R}^{\mathbf{L}} S\right) .
\end{aligned}
$$

Therefore $\operatorname{pd}_{S}\left(\mathbf{R H o m}_{S}\left(C \otimes_{R}^{\mathbf{L}} S, X \otimes_{R}^{\mathbf{L}} S\right)\right) \leq n$. Thus, we have

$$
\mathcal{P}_{C \otimes_{R}^{\mathrm{L}} S^{-}} \operatorname{pd}_{S}\left(X \otimes_{R}^{\mathrm{L}} S\right)-\sup \left(C \otimes_{R}^{\mathbf{L}} S\right) \leq n=\mathcal{P}_{C^{-}} \operatorname{pd}_{R}(X)-\sup (C),
$$

that is, the inequality in (i) holds.

Observe that since $\operatorname{fd}_{R}(S)<\infty$, we have $S \in \mathcal{A}_{C}(R)$, and hence $\sup \left(C \otimes_{R}^{\mathrm{L}} S\right) \leq \sup (C)$ by [2, Proposition 4.8(a)]. Hence, the inequality in (iii) follows from part (i). 
Now assume that $\varphi$ is faithfully flat. Therefore, one has that $\sup \left(C \otimes_{R}^{\mathbf{L}} S\right)=\sup (C)$. It suffices to show that $\mathcal{P}_{C \otimes_{R}^{\mathbf{L}} S^{-}} \operatorname{pd}_{R}\left(X \otimes_{R}^{\mathbf{L}} S\right) \geq$ $\mathcal{P}_{C^{-}} \operatorname{pd}_{R}(X)$. Assume that $\mathcal{P}_{C \otimes}{ }_{R} S^{-} \operatorname{pd}_{R}\left(X \otimes_{R}^{\mathrm{L}} S\right)=n<\infty$. Then

$$
\begin{aligned}
\operatorname{pd}_{S}\left(\mathbf{R H o m}_{R}(C, X) \otimes_{R}^{\mathbf{L}} S\right) & =\operatorname{pd}_{S}\left(\mathbf{R H o m}_{S}\left(C \otimes_{R}^{\mathbf{L}} S, X \otimes_{R}^{\mathbf{L}} S\right)\right) \\
& =n-\sup \left(C \otimes_{R}^{\mathbf{L}} S\right) .
\end{aligned}
$$

Therefore, we have $\operatorname{pd}_{S}\left(\mathbf{R H o m}_{R}(C, X) \otimes_{R}^{\mathbf{L}} S\right) \leq n-\sup (C)$. Observe that, if $P$ is an $R$-module such that $P \otimes_{R} S$ is projective over $S$, then $P$ is projective over $R$ by [7, Theorem 9.6] and [8]. A standard truncation argument thus shows that

$$
\operatorname{pd}_{R}\left(\mathbf{R} \operatorname{Hom}_{R}(C, X)\right) \leq \operatorname{pd}_{S}\left(\mathbf{R H o m}_{R}(C, X) \otimes_{R}^{\mathbf{L}} S\right)=n-\sup (C),
$$

as desired.

Parts (iv) and (ii) are proven similarly.

Corollary 3.12. Let $X \in \mathcal{D}_{\mathrm{b}}(R)$, and let $U \subset R$ be a multiplicatively closed subset. Then there are inequalities

(i) $\mathcal{P}_{U^{-1} C^{-}}-\operatorname{pd}_{U^{-1} R}\left(U^{-1} X\right) \leq \mathcal{P}_{C}-\operatorname{pd}_{R}(X)$,

(ii) $\mathcal{F}_{U^{-1} C^{-}} \operatorname{pd}_{U^{-1} R}\left(U^{-1} X\right) \leq \mathcal{F}_{C^{-}}-\operatorname{pd}_{R}(X)$,

(iii) $\mathcal{I}_{U^{-1} C^{-}} \operatorname{id}_{U^{-1} R}\left(U^{-1} X\right) \leq \mathcal{I}_{C}-\operatorname{id}_{R}(X)$,

(iv) $\mathcal{P}_{U^{-1} C^{-}}-\operatorname{pd}_{U^{-1} R}\left(U^{-1} X\right)-\sup \left(U^{-1} C\right) \leq \mathcal{P}_{C}-\operatorname{pd}_{R}(X)-\sup (C)$,

(v) $\mathcal{F}_{U^{-1} C^{-}}-\operatorname{pd}_{U^{-1} R}\left(U^{-1} X\right)-\sup \left(U^{-1} C\right) \leq \mathcal{F}_{C}-\operatorname{pd}_{R}(X)-\sup (C)$, and

(vi) $\mathcal{I}_{U^{-1} C^{-}} \operatorname{id}_{U^{-1} R}\left(U^{-1} X\right)-\sup \left(U^{-1} C\right) \leq \mathcal{I}_{C^{-}} \operatorname{id}_{R}(X)-\sup (C)$.

Proof. The map $\varphi: R \rightarrow U^{-1} R$ is flat. Hence, (i), (ii), (iv) and (v) follow from Proposition 3.11. Parts (iii) and (vi) are proven similarly to Proposition 3.11.

Remark 3.13. Observe that, to obtain the inequality in Corollary 3.12 , we need the inequality $\sup \left(U^{-1} C\right) \leq \sup (C)$ to hold. If we had defined $\mathcal{P}_{C}-\operatorname{pd}_{R}(X)$ as $\inf (C)+\operatorname{pd}_{R}\left(\mathbf{R} \operatorname{Hom}_{R}(C, X)\right)$, then Corollary 3.12 would not hold because

$$
\inf \left(U^{-1} C\right) \not \leq \inf (C) .
$$

This is why we choose $\sup (C)$ instead of $\inf (C)$ in the definition of $\mathcal{P}_{C^{-}}$pd. 
The next result is a local-global principal for Bass classes.

Lemma 3.14. Let $X \in \mathcal{D}_{\mathrm{b}}(R)$. The following conditions are equivalent:

(i) $X \in \mathcal{B}_{C}(R)$;

(ii) $U^{-1} X \in \mathcal{B}_{U^{-1} C}\left(U^{-1} R\right)$ for all multiplicatively closed subsets $U \subset R$

(iii) $X_{\mathfrak{p}} \in \mathcal{B}_{C_{\mathfrak{p}}}\left(R_{\mathfrak{p}}\right)$ for all $\mathfrak{p} \in \operatorname{Spec}(R)$;

(iv) $X_{\mathfrak{p}} \in \mathcal{B}_{C_{\mathfrak{p}}}\left(R_{\mathfrak{p}}\right)$ for all $\mathfrak{p} \in \operatorname{Supp}(R)$;

(v) $X_{\mathfrak{m}} \in \mathcal{B}_{C_{\mathfrak{m}}}\left(R_{\mathfrak{m}}\right)$ for all $\mathfrak{m} \in \operatorname{Max}(R)$; and

(vi) $X_{\mathfrak{m}} \in \mathcal{B}_{C_{\mathfrak{m}}}\left(R_{\mathfrak{m}}\right)$ for all $\mathfrak{m} \in \operatorname{Supp}(R) \cap \operatorname{Max}(R)$.

Proof. The implications (i) $\Rightarrow$ (ii) $\Rightarrow$ (iii) $\Rightarrow$ (iv) $\Rightarrow$ (vi) and (iii) $\Rightarrow$ (v) $\Rightarrow$ (vi) follow from definitions. We prove (v) $\Rightarrow$ (i) and (vi) $\Rightarrow$ (v).

For the implication $(\mathrm{v}) \Rightarrow$ (i), assume $X_{\mathfrak{m}} \in \mathcal{B}_{C_{\mathfrak{m}}}\left(R_{\mathfrak{m}}\right)$ for all $\mathfrak{m} \in \operatorname{Max}(R)$. We use the following commutative diagram in $\mathcal{D}(R)$ :

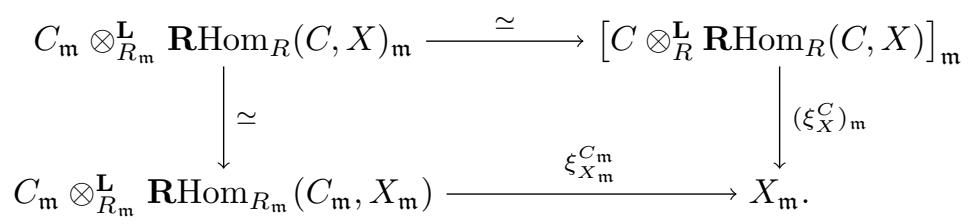

As $X_{\mathfrak{m}} \in \mathcal{B}_{C_{\mathfrak{m}}}\left(R_{\mathfrak{m}}\right)$ for all $\mathfrak{m} \in \operatorname{Max}(R)$, the morphism $\xi_{X_{\mathfrak{m}}}^{C_{\mathfrak{m}}}$ is an isomorphism for all $\mathfrak{m} \in \operatorname{Max}(R)$. Commutativity of the above diagram now forces $\left(\xi_{X}^{C}\right)_{\mathfrak{m}}$ to be an isomorphism for all $\mathfrak{m} \in \operatorname{Max}(R)$. Therefore, $\xi_{X}^{C}$ is an isomorphism.

It remains to show that $\mathbf{R} \operatorname{Hom}_{R}(C, X) \in \mathcal{D}_{\mathrm{b}}(R)$. As $\mathbf{R} \operatorname{Hom}_{R}(C, X) \in$ $\mathcal{D}_{-}(R)$, it suffices to show that $\mathbf{R H o m}_{R}(C, X) \in \mathcal{D}_{+}(R)$. By assumption, $X_{\mathfrak{m}} \in \mathcal{B}_{C_{\mathfrak{m}}}\left(R_{\mathfrak{m}}\right)$. Then, for all $\mathfrak{m} \in \operatorname{Max}(R)$, we have

$$
\begin{aligned}
\inf \left(\mathbf{R H o m}_{R}(C, X)_{\mathfrak{m}}\right) & =\inf \left(\mathbf{R} \operatorname{Hom}_{R_{\mathfrak{m}}}\left(C_{\mathfrak{m}}, X_{\mathfrak{m}}\right)\right) \\
& \geq \inf \left(X_{\mathfrak{m}}\right)-\sup \left(C_{\mathfrak{m}}\right) \\
& \geq \inf (X)-\sup (C),
\end{aligned}
$$


where the isomorphism $\mathbf{R H o m}_{R}(C, X)_{\mathfrak{m}} \simeq \mathbf{R H o m}_{R_{\mathfrak{m}}}\left(C_{\mathfrak{m}}, X_{\mathfrak{m}}\right)$ gives us the equality, the first inequality is by [2, Proposition 4.8(c)] and the second inequality is by properties of localization. Thus, $\inf \left(\mathbf{R H o m}_{R}(C, X)\right) \geq \inf (X)-\sup (C)>-\infty$.

For the implication (vi) $\Rightarrow(\mathrm{v})$, assume $X_{\mathfrak{m}} \in \mathcal{B}_{C_{\mathfrak{m}}}\left(R_{\mathfrak{m}}\right)$ for all $\mathfrak{m} \in \operatorname{Supp}_{R}(X) \cap \operatorname{Max}(R)$. Then, for all $\mathfrak{m} \in \operatorname{Max}(R) \backslash \operatorname{Supp}_{R}(X)$, we have $X_{\mathfrak{m}} \simeq 0 \in \mathcal{B}_{C_{\mathfrak{m}}}\left(R_{\mathfrak{m}}\right)$, as desired.

The following is proven similarly to Lemma 3.14.

Lemma 3.15. Let $X \in \mathcal{D}_{\mathrm{b}}(R)$. The following conditions are equivalent:

(i) $X \in \mathcal{A}_{C}(R)$;

(ii) $U^{-1} X \in \mathcal{A}_{U^{-1} C}\left(U^{-1} R\right)$ for all multiplicatively closed subsets $U \subset R$

(iii) $X_{\mathfrak{p}} \in \mathcal{A}_{C_{\mathfrak{p}}}\left(R_{\mathfrak{p}}\right)$ for all $\mathfrak{p} \in \operatorname{Spec}(R)$;

(iv) $X_{\mathfrak{p}} \in \mathcal{A}_{C_{\mathfrak{p}}}\left(R_{\mathfrak{p}}\right)$ for all $\mathfrak{p} \in \operatorname{Supp}(R)$;

(v) $X_{\mathfrak{m}} \in \mathcal{A}_{C_{\mathfrak{m}}}\left(R_{\mathfrak{m}}\right)$ for all $\mathfrak{m} \in \operatorname{Max}(R)$; and

(vi) $X_{\mathfrak{m}} \in \mathcal{A}_{C_{\mathfrak{m}}}\left(R_{\mathfrak{m}}\right)$ for all $\mathfrak{m} \in \operatorname{Supp}(R) \cap \operatorname{Max}(R)$.

Proposition 3.16. Let $X \in \mathcal{D}_{\mathrm{b}}(R)$, and let $n \in \mathbb{Z}$. Consider the following conditions:

(i) $\mathcal{P}_{C}-\operatorname{pd}_{R}(X)-\sup (C) \leq n$;

(ii) $\mathcal{P}_{U^{-1} C^{-}} \operatorname{pd}_{U^{-1} R}\left(U^{-1} X\right)-\sup \left(U^{-1} C\right) \leq n$ for each multiplicatively closed subset $U \subset R$;

(iii) $\mathcal{P}_{C_{\mathfrak{p}}}-\operatorname{pd}_{R_{\mathfrak{p}}}\left(X_{\mathfrak{p}}\right)-\sup \left(C_{\mathfrak{p}}\right) \leq n$ for each $\mathfrak{p} \in \operatorname{Spec}(R)$; and

(iv) $\mathcal{P}_{C_{\mathfrak{m}}}-\operatorname{pd}_{R_{\mathfrak{m}}}\left(X_{\mathfrak{m}}\right)-\sup \left(C_{\mathfrak{m}}\right) \leq n$ for each $\mathfrak{m} \in \operatorname{Max}(R)$.

Then (i) $\Rightarrow$ (ii) $\Rightarrow$ (iii) $\Rightarrow$ (iv). Furthermore, if $X \in \mathcal{D}_{\mathrm{b}}^{\mathrm{f}}(R)$, then (iv) $\Rightarrow$ (i), and

$$
\begin{aligned}
& \mathcal{P}_{C}-\operatorname{pd}_{R}(X)-c=\sup \left\{\begin{array}{l|l}
\mathcal{P}_{U^{-1} C^{-}-\mathrm{pd}_{U-1}\left(U^{-1} X\right)} & \begin{array}{l}
U \subset R \text { is } \\
-\sup \left(U^{-1} C\right)
\end{array} \\
\begin{array}{l|l}
\text { multiplicatively } \\
\text { closed }
\end{array}
\end{array}\right\} \\
& =\sup \left\{\mathcal{P}_{C_{\mathfrak{p}}}-\operatorname{pd}_{R_{\mathfrak{p}}}\left(X_{\mathfrak{p}}\right)-\sup \left(C_{\mathfrak{p}}\right) \mid \mathfrak{p} \in \operatorname{Spec}(R)\right\} \\
& =\sup \left\{\mathcal{P}_{C_{\mathfrak{m}}}-\operatorname{pd}_{R_{\mathfrak{m}}}\left(X_{\mathfrak{m}}\right)-\sup \left(C_{\mathfrak{m}}\right) \mid \mathfrak{m} \in \operatorname{Max}(R)\right\},
\end{aligned}
$$

where $c=\sup (C)$. 
Proof. Observe that (i) $\Rightarrow$ (ii) follows from Proposition 3.11. The implications (ii) $\Rightarrow$ (iii) $\Rightarrow$ (iv) follow from properties of localization. For the rest of the proof, assume that $X \in \mathcal{D}_{\mathrm{b}}^{\mathrm{f}}(R)$.

For the implication (iv) $\Rightarrow$ (i), assume that $\mathcal{P}_{C_{\mathfrak{m}}}-\operatorname{pd}_{R_{\mathfrak{m}}}\left(X_{\mathfrak{m}}\right)-$ $\sup \left(C_{\mathfrak{m}}\right) \leq n<\infty$ for all $\mathfrak{m} \in \operatorname{Max}(R)$. Then, by Remark 3.2, we have $X_{\mathfrak{m}} \in \mathcal{B}_{C_{\mathfrak{m}}}\left(R_{\mathfrak{m}}\right)$ for all $\mathfrak{m} \in \operatorname{Max}(R)$. Therefore, Lemma 3.14 implies that $X \in \mathcal{B}_{C}(R)$, and hence, $\mathbf{R H o m}_{R}(C, X) \in \mathcal{D}_{\mathrm{b}}(R)$. Now

$$
\begin{aligned}
\mathcal{P}_{C^{-}} \operatorname{pd}_{R}(X)-\sup (C) & =\operatorname{pd}_{R}\left(\mathbf{R} \operatorname{Hom}_{R}(C, X)\right) \\
& =\sup _{\mathfrak{m} \in \operatorname{Max}(R)}\left(\operatorname{pd}_{R_{\mathfrak{m}}}\left(\mathbf{R H o m} R_{\mathfrak{m}}\left(C_{\mathfrak{m}}, X_{\mathfrak{m}}\right)\right)\right) \\
& \leq n,
\end{aligned}
$$

where the second equality is by [1, Proposition 5.3P].

For the equalities, assume first that $\mathcal{P}_{C^{-}} \operatorname{pd}_{R}(X)-\sup (C)=n<\infty$. Then, each displayed supremum in the statement is at most $n$. If any of the supremums are strictly less than $n$, then the above equivalence will force $\mathcal{P}_{C^{-}} \operatorname{pd}_{R}(X)-\sup (C)<n$, contradicting our assumption. A similar argument establishes the desired equalities if we assume any of the supremums equal $n$.

Finally, if any of the displayed values in the statement are infinite, then the above equivalences forces the other values to be infinite as well.

To prove the implication (iv) $\Rightarrow$ (i) in Proposition 3.16, the condition $X \in \mathcal{D}_{\mathrm{b}}^{\mathrm{f}}(R)$ is required. However, the flat and injective versions only require $X \in \mathcal{D}_{\mathrm{b}}(R)$; see [1, Propositions 5.3F, 5.3I]. Thus, the next two results are proven similarly to Proposition 3.16.

Proposition 3.17. Let $X \in \mathcal{D}_{\mathrm{b}}(R)$, and let $n \in \mathbb{Z}$. The following conditions are equivalent:

(i) $\mathcal{F}_{C}-\operatorname{pd}_{R}(X)-\sup (C) \leq n$;

(ii) $\mathcal{F}_{U^{-1} C^{-}} \operatorname{pd}_{U^{-1} R}\left(U^{-1} X\right)-\sup \left(U^{-1} C\right) \leq n$ for each multiplicatively closed subset $U \subset R$;

(iii) $\mathcal{F}_{C_{\mathfrak{p}}}-\operatorname{pd}_{R_{\mathfrak{p}}}\left(X_{\mathfrak{p}}\right)-\sup \left(C_{\mathfrak{p}}\right) \leq n$ for each prime ideal $\mathfrak{p} \subset R$; and

(iv) $\mathcal{F}_{C_{\mathfrak{m}}}-\operatorname{pd}_{R_{\mathfrak{m}}}\left(X_{\mathfrak{m}}\right)-\sup \left(C_{\mathfrak{m}}\right) \leq n$ for each maximal ideal $\mathfrak{m} \subset R$. 
Furthermore,

$$
\begin{aligned}
\mathcal{F}_{C}-\operatorname{pd}_{R}(X)-c & =\sup \left\{\begin{array}{l|l}
\mathcal{F}_{U^{-1} C^{-}-\operatorname{pd}_{U^{-1} R}\left(U^{-1} X\right)} & \begin{array}{l}
U \subset R \text { is } \\
-\sup \left(U^{-1} C\right) \\
\text { multiplicatively } \\
\text { closed }
\end{array}
\end{array}\right\} \\
& =\sup \left\{\mathcal{F}_{C_{\mathfrak{p}}}-\operatorname{pd}_{R_{\mathfrak{p}}}\left(X_{\mathfrak{p}}\right)-\sup \left(C_{\mathfrak{p}}\right) \mid \mathfrak{p} \in \operatorname{Spec}(R)\right\} \\
& =\sup \left\{\mathcal{F}_{C_{\mathfrak{m}}}-\operatorname{pd}_{R_{\mathfrak{m}}}\left(X_{\mathfrak{m}}\right)-\sup \left(C_{\mathfrak{m}}\right) \mid \mathfrak{m} \in \operatorname{Max}(R)\right\},
\end{aligned}
$$

where $c=\sup (C)$.

Proposition 3.18. Let $X \in \mathcal{D}_{\mathrm{b}}(R)$, and let $n \in \mathbb{Z}$. The following conditions are equivalent:

(i) $\mathcal{I}_{C}-\mathrm{id}_{R}(X)-\sup (C) \leq n$;

(ii) $\mathcal{I}_{U^{-1} C^{-}} \operatorname{id}_{U^{-1} R}\left(U^{-1} X\right)-\sup \left(U^{-1} C\right) \leq n$ for each multiplicatively closed subset $U \subset R$;

(iii) $\mathcal{I}_{C_{\mathfrak{p}}}-\operatorname{id}_{R_{\mathfrak{p}}}\left(X_{\mathfrak{p}}\right)-\sup \left(C_{\mathfrak{p}}\right) \leq n$ for each prime ideal $\mathfrak{p} \subset R$; and

(iv) $\mathcal{I}_{C_{\mathfrak{m}}}-\operatorname{id}_{R_{\mathfrak{m}}}\left(X_{\mathfrak{m}}\right)-\sup \left(C_{\mathfrak{m}}\right) \leq n$ for each maximal ideal $\mathfrak{m} \subset R$.

Furthermore,

$$
\begin{aligned}
\mathcal{I}_{C^{-}-\operatorname{id}_{R}(X)-c} & =\sup \left\{\begin{array}{l|l}
\operatorname{id}_{U^{-1} R}\left(U^{-1} C \otimes_{U^{-1} R}^{\mathbf{L}} U^{-1} X\right) & \begin{array}{l}
U \subset R \text { is } \\
-\sup \left(U^{-1} C\right) \\
\text { multiplicatively } \\
\text { closed }
\end{array}
\end{array}\right\} \\
& =\sup \left\{\operatorname{id}_{R_{\mathfrak{p}}}-\operatorname{id}_{R_{\mathfrak{p}}}\left(C_{\mathfrak{p}} \otimes_{R_{\mathfrak{p}}}^{\mathbf{L}} X_{\mathfrak{p}}\right)-\sup \left(C_{\mathfrak{m}}\right) \mid \mathfrak{p} \in \operatorname{Spec}(R)\right\} \\
& =\sup \left\{\operatorname{id}_{R_{\mathfrak{m}}}-\operatorname{id}_{R_{\mathfrak{m}}}\left(C_{\mathfrak{m}} \otimes_{R_{\mathfrak{m}}}^{\mathbf{L}} X_{\mathfrak{m}}\right)-\sup \left(C_{\mathfrak{m}}\right) \mid \mathfrak{m} \in \operatorname{Max}(R)\right\},
\end{aligned}
$$

where $c=\sup (C)$.

Remark 3.19. When $C$ is a semidualizing $R$-module, e.g., $C=R$, we recover the known local-global conditions for $\mathcal{P}_{C^{-}}$pd, $\mathcal{F}_{C^{-}}$pd, $\mathcal{I}_{C^{-}}$id, pd, fd, and id.

4. Stability results. In this section, we investigate the behavior of $\mathcal{P}_{C^{-}}$pd, $\mathcal{F}_{C^{-}}$pd and $\mathcal{I}_{C^{-}}$id after applying the functors $\otimes^{\mathbf{L}}$ and $\mathbf{R H o m}$.

Proposition 4.1. Let $X, Y \in \mathcal{D}_{\mathrm{b}}(R)$. The following inequalities hold:

(i) $\mathcal{P}_{C}-\operatorname{pd}_{R}\left(X \otimes_{R}^{\mathrm{L}} Y\right) \leq \mathcal{P}_{C}-\operatorname{pd}_{R}(X)+\operatorname{pd}_{R}(Y)$;

(ii) $\mathcal{I}_{C}-\operatorname{id}_{R}\left(\mathbf{R} \operatorname{Hom}_{R}(X, Y)\right) \leq \mathcal{F}_{C}-\operatorname{pd}_{R}(X)+\operatorname{id}_{R}(Y)$; and 
(iii) $\mathcal{F}_{C}-\operatorname{pd}_{R}\left(X \otimes_{R}^{\mathbf{L}} Y\right) \leq \mathcal{F}_{C}-\operatorname{pd}_{R}(X)+\operatorname{fd}_{R}(Y)$.

Proof.

(i) Without loss of generality, we assume that $\mathcal{P}_{C^{-}} \operatorname{pd}_{R}(X)<\infty$ and $\operatorname{pd}_{R}(Y)<\infty$. It now follows that $\mathcal{P}_{C^{-}} \operatorname{pd}_{R}(X)=\sup (C)+$ $\operatorname{pd}_{R}\left(\mathbf{R} \operatorname{Hom}_{R}(C, X)\right)$. By [1, Theorem $\left.4.1(\mathrm{P})\right]$, we have that

$$
\operatorname{pd}_{R}\left(\mathbf{R H o m}_{R}(C, X) \otimes_{R}^{\mathbf{L}} Y\right) \leq \operatorname{pd}_{R}\left(\mathbf{R H o m}_{R}(C, X)\right)+\operatorname{pd}_{R}(Y) .
$$

Since $\operatorname{pd}_{R}(Y)<\infty$ (hence $\operatorname{fd}_{R}(Y)<\infty$ ) we get that the tensorevaluation (2.4) is an isomorphism in $\mathcal{D}(R)$. That is, $\mathbf{R H o m}_{R}\left(C, X \otimes_{R}^{\mathbf{L}}\right.$ $Y) \simeq \mathbf{R H o m}_{R}(C, X) \otimes_{R}^{\mathbf{L}} Y$. Hence, we have

$$
\operatorname{pd}_{R}\left(\mathbf{R} \operatorname{Hom}_{R}\left(C, X \otimes_{R}^{\mathbf{L}} Y\right)\right) \leq \operatorname{pd}_{R}\left(\mathbf{R} \operatorname{Hom}_{R}(C, X)\right)+\operatorname{pd}_{R}(Y) .
$$

By adding $\sup (C)$ to each side we see that $\mathcal{P}_{C^{-}} \operatorname{pd}_{R}\left(X \otimes_{R}^{\mathbf{L}} Y\right) \leq$ $\mathcal{P}_{C}-\operatorname{pd}_{R}(X)+\operatorname{pd}_{R}(Y)$.

(ii) and (ii) are proven similarly to (i).

Corollary 4.2. Let $X \in \mathcal{D}_{b}(R)$. The following inequalities hold:

(i) $\mathcal{P}_{C}-\operatorname{pd}_{R}\left(X \otimes_{R}^{\mathbf{L}} \mathbf{R} \operatorname{Hom}_{R}(C, Y)\right) \leq \mathcal{P}_{C}-\operatorname{pd}_{R}(X)+\mathcal{P}_{C}-\operatorname{pd}_{R}(Y)-$ $\sup (C)$

(ii) $\mathcal{I}_{C}-\operatorname{id}_{R}\left(\mathbf{R H o m}_{R}\left(X, C \otimes_{R}^{\mathrm{L}} Y\right)\right) \leq \mathcal{F}_{C}-\operatorname{pd}_{R}(X)+\mathcal{I}_{C}-\operatorname{id}_{R}(Y)-$ $\sup (C) ;$ and

(iii) $\mathcal{F}_{C}-\operatorname{pd}_{R}\left(X \otimes_{R}^{\mathbf{L}} \mathbf{R} \operatorname{Hom}_{R}(C, Y)\right) \leq \mathcal{F}_{C}-\operatorname{pd}(R X)+\mathcal{F}_{C}-\operatorname{pd}_{R}(Y)-$ $\sup (C)$.

Proof.

(i). By Proposition 4.1 (i), we have that $\mathcal{P}_{C^{-}} \operatorname{pd}_{R}\left(X \otimes_{R}^{\mathbf{L}} \mathbf{R} \operatorname{Hom}_{R}(C, Y)\right)$ $\leq \mathcal{P}_{C^{-}} \operatorname{pd}_{R}(X)+\operatorname{pd}_{R}\left(\mathbf{R H o m}{ }_{R}(C, Y)\right)$. Add and subtract $\sup (C)$ to the right hand side to obtain the result.

(ii) and (iii) are proven similarly.

The next result is a version of Fact 2.2 involving a semidualizing complex.

Proposition 4.3. Let $X, Y \in \mathcal{D}_{\mathrm{b}}(R)$. 
(i) If $\operatorname{id}_{R}(Y)<\infty$, then $\mathcal{F}_{C}-\operatorname{pd}_{R}\left(\mathbf{R H o m}_{R}(X, Y)\right) \leq \mathcal{I}_{C}-\operatorname{id}_{R}(X)+$ $\sup (Y)$.

(ii) If $\operatorname{fd}_{R}(Y)<\infty$, then $\mathcal{I}_{C}-\operatorname{id}_{R}\left(X \otimes_{R}^{\mathbf{L}} Y\right) \leq \mathcal{I}_{C^{-}} \operatorname{id}_{R}(X)-\inf (Y)$.

Proof. that

(i) Assume that $\operatorname{id}_{R}(Y)<\infty$. By applying Definition 3.1, we get $\mathcal{F}_{C^{-}} \operatorname{pd}_{R}\left(\mathbf{R H o m}_{R}(X, Y)\right)=\sup (C)+\operatorname{fd}_{R}\left(\mathbf{R H o m}_{R}\left(C, \operatorname{RHom}_{R}(X, Y)\right)\right)$.

Observe that, by Hom-tensor adjointness, there is an isomorphism

$$
\mathbf{R H o m}_{R}\left(C, \mathbf{R H o m}_{R}(X, Y)\right) \simeq \mathbf{R H o m}_{R}\left(C \otimes \otimes_{R}^{\mathbf{L}} X, Y\right) .
$$

Therefore, $\operatorname{fd}_{R}\left(\mathbf{R H o m}_{R}\left(C, \mathbf{R H o m}_{R}(X, Y)\right)\right)=\mathrm{fd}_{R}\left(\mathbf{R H o m}_{R}\left(C \otimes \mathbf{L}_{R} X, Y\right)\right)$. Hence, by Fact 2.2 (a), we have that

$$
\mathrm{fd}_{R}\left(\mathbf{R} \operatorname{Hom}_{R}\left(C \otimes_{R}^{\mathbf{L}} X, Y\right)\right) \leq \operatorname{id}_{R}\left(C \otimes_{R}^{\mathbf{L}} X\right)+\sup (Y) .
$$

By adding $\sup (C)$ to each side of the above inequality, we obtain the desired result.

(ii) is proven similarly.

Proposition 4.4. Let $X \in \mathcal{D}_{\mathrm{b}}(R)$. The following conditions are equivalent:

(i) $\mathcal{F}_{C}-\operatorname{pd}_{R}(X)<\infty$;

(ii) $\mathcal{I}_{C^{-}} \mathrm{id}_{R}\left(\mathbf{R H o m}_{R}(X, Y)\right)<\infty$ for all $Y \in \mathcal{D}_{\mathrm{b}}(R)$ such that $\operatorname{id}_{R}(Y)<\infty ;$ and

(iii) $\mathcal{I}_{C}-\operatorname{id}_{R}\left(\mathbf{R H o m}_{R}(X, E)\right)<\infty$ for some faithfully injective $R$ module $E$.

Proof. (i) $\Rightarrow$ (ii). This follows from Proposition 4.1 (ii).

(ii) $\Rightarrow$ (iii). Since $E$ is a faithfully injective module we have $\operatorname{id}_{R}(E)=$ $0<\infty$. Therefore, (ii) implies that $\mathcal{I}_{C^{-}} \operatorname{id}_{R}\left(\mathbf{R H o m}_{R}(X, E)\right)<\infty$.

(iii) $\Rightarrow$ (i). Assume that there exists a faithfully injective $R$-module $E$ such that $\mathcal{I}_{C}-\mathrm{id}_{R}\left(\mathbf{R H o m}_{R}(X, E)\right)<\infty$. Then, by Definition 3.1 (iii), $\mathcal{I}_{C^{-}} \operatorname{id}_{R}\left(\mathbf{R H o m}_{R}(X, E)\right)=\sup (C)+\operatorname{id}_{R}\left(C \otimes_{R}^{\mathbf{L}} \mathbf{R H o m}_{R}(X, E)\right)$. By Hom-evaluation (2.4), there is an isomorphism

$$
\mathbf{R H o m}_{R}\left(\mathbf{R H o m}_{R}(C, X), E\right) \simeq C \otimes_{R}^{\mathbf{L}} \mathbf{R H o m}_{R}(X, E) .
$$


It follows that

$$
\operatorname{id}_{R}\left(C \otimes \otimes_{R}^{\mathbf{L}} \mathbf{R H o m}_{R}(X, E)\right)=\operatorname{id}_{R}\left(\mathbf{R H o m}_{R}\left(\mathbf{R H o m}_{R}(C, X), E\right)\right)<\infty .
$$

Therefore, by Lemma 2.3, $\mathrm{fd}_{R}\left(\mathbf{R H o m}_{R}(C, X)\right)<\infty$. It now follows that $\mathcal{F}_{C^{-}} \operatorname{pd}_{R}(X)<\infty$.

The following three propositions are proven similarly to Proposition 4.4.

Proposition 4.5. Let $X \in \mathcal{D}_{\mathrm{b}}(R)$. The following conditions are equivalent:

(i) $\mathcal{F}_{C}-\operatorname{pd}_{R}(X)<\infty$;

(ii) $\mathcal{F}_{C}-\operatorname{pd}_{R}\left(X \otimes{ }_{R}^{\mathbf{L}} Y\right)<\infty$ for all $Y \in \mathcal{D}_{\mathrm{b}}(R)$ such that $\operatorname{fd}_{R}(Y)<\infty$;

(iii) $\mathcal{F}_{C}-\operatorname{pd}_{R}\left(X \otimes_{R}^{\mathbf{L}} F\right)<\infty$ for some faithfully flat $R$-module $F$.

Proposition 4.6. Let $X \in \mathcal{D}_{\mathrm{b}}(R)$. The following conditions are equivalent:

(i) $\mathcal{I}_{C}-\mathrm{id}_{R}(X)<\infty$;

(ii) $\mathcal{F}_{C}-\operatorname{pd}_{R}\left(\mathbf{R H o m}_{R}(X, Y)\right)<\infty$ for all $Y \in \mathcal{D}_{\mathrm{b}}(R)$ such that $\operatorname{id}_{R}(Y)<\infty$

(iii) $\mathcal{F}_{C}-\operatorname{pd}_{R}\left(\mathbf{R H o m}_{R}(X, E)\right)<\infty$ for some faithfully injective $R$ module $E$.

Proposition 4.7. Let $X \in \mathcal{D}_{\mathrm{b}}(R)$. The following conditions are equivalent:

(i) $\mathcal{I}_{C}-\mathrm{id}_{R}(X)<\infty$;

(ii) $\mathcal{I}_{C}-\operatorname{id}_{R}\left(X \otimes_{R}^{\mathbf{L}} Y\right)<\infty$ for all $Y \in \mathcal{D}_{\mathrm{b}}(R)$ such that $\operatorname{fd}_{R}(Y)<\infty$;

(iii) $\mathcal{I}_{C}-\operatorname{id}_{R}\left(X \otimes \otimes_{R}^{\mathbf{L}} F\right)<\infty$ for some faithfully flat $R$-module $F$.

Corollary 4.8. Let $X \in \mathcal{D}_{\mathrm{b}}(R)$ and, if there exists a dualizing complex $D$ and $\mathcal{F}_{C}-\operatorname{pd}_{R}(X)<\infty$, then $\mathcal{I}_{C}-\mathrm{id}_{R}\left(X^{\dagger}\right)<\infty$ where $X^{\dagger}=\mathbf{R H o m}_{R}(X, D)$.

Proof. Since $D$ is a dualizing complex, it has finite injective dimension. Therefore, the result follows from Proposition 4.4. 
The last result of this paper establishes Theorem 1.2 from the introduction.

Theorem 4.9. Assume $R$ has a dualizing complex $D$, and let $X \in$ $\mathcal{D}_{\mathrm{b}}(R)$. Then $\mathcal{I}_{C}-\mathrm{id}_{R}(X)<\infty$ if and only if $\mathcal{F}_{C^{\dagger}}-\operatorname{pd}_{R}(X)<\infty$ where $C^{\dagger}=\mathbf{R H o m}_{R}(C, D)$.

Proof. For the forward implication, assume that $\mathcal{I}_{C^{-}} \operatorname{id}_{R}(X)<\infty$. Then, set $J=C \otimes_{R}^{\mathbf{L}} X$. Since $\mathcal{I}_{C^{-}} \operatorname{id}_{R}(X)<\infty$ we have that $J$ has finite injective dimension. By Remark 3.2, we have $X \in \mathcal{A}_{C}(R)$. This explains the first isomorphism in the following display:

$$
\begin{aligned}
X & \simeq \mathbf{R H o m}_{R}(C, J) \simeq \mathbf{R H o m}_{R}\left(\mathbf{R H o m}_{R}\left(C^{\dagger}, D\right), J\right) \\
& \simeq C^{\dagger} \otimes_{R}^{\mathbf{L}} \mathbf{R H o m}_{R}(D, J) .
\end{aligned}
$$

The second isomorphism is from the isomorphism $C \simeq C^{\dagger \dagger}$, and the

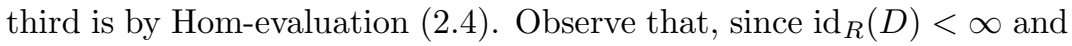
$\operatorname{id}_{R}(J)<\infty$, we have $\operatorname{fd}_{R}\left(\mathbf{R H o m}_{R}(D, J)\right)<\infty$ by Fact 2.2 (a). Thus, it follows that $\mathcal{F}_{C^{\dagger}}-\mathrm{fd}_{R}(X)<\infty$ by the displayed isomorphisms.

For the reverse implication, assume that $\mathcal{F}_{C^{\dagger}}-\mathrm{fd}_{R}(X)<\infty$. Then we can write $X \simeq C^{\dagger} \otimes_{R}^{\mathbf{L}} F$, where $F=\mathbf{R H o m}_{R}\left(C^{\dagger}, X\right)$ and $\operatorname{fd}_{R}(F)<\infty$. We then have the following isomorphisms:

$$
X \simeq C^{\dagger} \otimes_{R}^{\mathbf{L}} F=\mathbf{R H o m}_{R}(C, D) \otimes_{R}^{\mathbf{L}} F \simeq \mathbf{R H o m}_{R}\left(C, D \otimes_{R}^{\mathbf{L}} F\right),
$$

where the second isomorphism is by tensor-evaluation (2.4). Since $\operatorname{id}_{R}(D)<\infty$ and $\operatorname{fd}_{R}(F)<\infty$, we have $\operatorname{id}_{R}\left(D \otimes_{R}^{\mathbf{L}} F\right)<\infty$ by Fact 2.2 (b). Hence, $\mathcal{I}_{C^{-}} \mathrm{id}_{R}(X)<\infty$ by Theorem 3.9 (iii), as desired.

Acknowledgments. I am grateful to the referee for thoughtful comments and to my advisor Sean Sather-Wagstaff for all of his guidance and advice.

\section{REFERENCES}

1. L.L. Avramov and H.-B. Foxby, Homological dimensions of unbounded complexes, J. Pure Appl. Algebra 71 (1991), 129-155.

2. L.W. Christensen, Semi-dualizing complexes and their Auslander categories, Trans. Amer. Math. Soc. 353 (2001), 1839-1883.

3. H.-B. Foxby, Bounded complexes of flat modules, J. Pure Appl. Algebra 15 (1979), 149-172. 
4. A. Frankild and S. Sather-Wagstaff. Reflexivity and ring homomorphisms of finite flat dimension, Comm. Algebra 35 (2007), 461-500.

5. S.I. Gelfand and Y.I. Manin, Methods of homological algebra, Springer-Verlag, Berlin, 1996.

6. R. Hartshorne, Residues and duality, Lect. Notes Math. 20, Springer-Verlag, Berlin, 1966.

7. A. Perry, Faithfully flat descent for projectivity of modules, ArXiv e-prints, October 2010.

8. M. Raynaud and L. Gruson, Critères de platitude et de projectivité, Techniques de "platification" d'un module, Invent. Math. 13 (1971), 1-89.

9. Sean Sather-Wagstaff and Jonathan Totushek, Using semidualizing complexes to detect Gorenstein rings, Arch. Math. 104 (2015), 523-529.

10. R. Takahashi and D. White, Homological aspects of semidualizing modules, Math. Scand. 106 (2010), 5-22.

Mathematics and Computer Science Department, University of WisconsinSuperior, Swenson Hall 3030, Belknap and Catlin Ave, P.O. Box 2000, SupERIOR, WI 54880

Email address: jtotushe@uwsuper.edu 